DRAFT VERSION OCTOBER 31, 2018

Preprint typeset using LATEX style emulateapj v. 08/22/09

\title{
PROMPT SHOCKS IN THE GAS DISK AROUND A RECOILING SUPERMASSIVE BLACK HOLE BINARY
}

\author{
ZOLTÁN LIPPAI, ZSOLT FREI \\ Institute of Physics, Eötvös University, Pázmány P. s. 1/A, 1117 Budapest, Hungary \\ ZOLTÁN HAIMAN \\ Department of Astronomy, Columbia University, 550 West 120th Street, New York, NY 10027, USA \\ Draft version October 31, 2018
}

\begin{abstract}
Supermassive black hole binaries (BHBs) produced in galaxy mergers recoil at the time of their coalescence due to the emission of gravitational waves (GWs). We simulate the response of a thin, two-dimensional disk of collisionless particles, initially on circular orbits around a $10^{6} \mathrm{M}_{\odot}$ BHB, to kicks that are either parallel or perpendicular to the initial orbital plane. Typical kick velocities $\left(v_{\text {kick }}\right)$ can exceed the sound speed in a circumbinary gas disk. While the inner disk is strongly bound to the recoiling binary, the outer disk is only weakly bound or unbound. This leads to differential motions in the disturbed disk that increase with radius and can become supersonic at $\gtrsim 700$ Schwarzschild radii for $v_{\text {kick }}=500 \mathrm{~km} \mathrm{~s}^{-1}$, implying that shocks form beyond this radius. We indeed find that kicks in the disk plane lead to immediate strong density enhancements (within weeks) in a tightly wound spiral caustic, propagating outward at the speed $\sim v_{\text {kick}}$. Concentric density enhancements are also observed for kicks perpendicular to the disk, but are weaker and develop into caustics only after a long delay (>one year). Unless both BH spins are low or precisely aligned with the orbital angular momentum, a significant fraction ( plane for strong shocks to be produced. The shocks could result in an afterglow whose characteristic photon energy increases with time, from the $\mathrm{UV}(\sim 10 \mathrm{eV})$ to the soft $\mathrm{X}$-ray $(\sim 100 \mathrm{eV})$ range, between one month and one year after the merger. This could help identify EM counterparts to GW sources discovered by LISA.
\end{abstract}

Subject headings: black hole physics - galaxies: nuclei - gravitational waves

\section{INTRODUCTION}

The recent break-through in numerical relativity has allowed a direct computation of the linear momentum flux produced during the coalescence of a $\mathrm{BH}$ binary (Baker et al. 2006, 2007; Campanelli et al. 2007a,b; González et al. 2007a,b; Herrmann et al. 2007a,c; Koppitz et al. 2007). The resulting final recoil depends on the masses, orbital parameters, and spins of the BHs, and in special configurations (i.e. with spins anti-aligned with each other), it can reach velocities as high as $\approx 4,000 \mathrm{~km} \mathrm{~s}^{-1}$ (González 2007b). Much of the recent literature focused on the astrophysical implications of high-velocity kicks, which may displace or remove supermassive BHs from galactic centers (e.g. Merritt et al. 2004; Madau \& Quataert 2004; Loeb 2007), and inhibit the growth of BHs at high redshifts $(z \gtrsim 6)$, where the escape velocities from low-mass galactic halos is small ( $\lesssim 100 \mathrm{~km} \mathrm{~s}^{-1}$; Haiman 2004, Yoo \& Miralda-Escude 2004, Shapiro 2005; Volonteri \& Perna 2005).

Another exciting implication of kicks is that they may help produce an electromagnetic (EM) counterpart of gravitational wave sources detected by the future Laser Interferometric Space Antenna (LISA) satellite. The discovery of such a counterpart would constitute a milestone for fundamental physics and astrophysics (e.g. Kocsis et al. 2007b). If the BHB is surrounded by a circumbinary gas disk, the disk will respond promptly (on the local orbital timescale) to such a kick. If this results in warps or shocks, the disturbed disk could produce a transient EM signature (Milosavljević \& Phinney 2005). The sky localization uncertainty from the LISA instrument several weeks prior to merger is typically a few square degrees (Kocsis et al. 2007a; Lang \& Hughes 2007), and the kick can begin building up at the end of the inspiral phase, before the final coalescence (Schnittman et al. 2007). This will make it possible, in many cases, to monitor a few square-degree area on the sky prior to, during, as well as immediately following the coalescence of BHs in the mass range $\sim\left(10^{5}-10^{7}\right) \mathrm{M}_{\odot} /(1+z)$ at redshifts out to $z \sim 3$, and search for a prompt transient signature associated with the kick (Kocsis et al. 2007b).

In the context of producing a prompt EM counterpart, we expect that the direction, in addition to the magnitude of the kick, will be important. Naively, one expects that a kick within the plane of any circumbinary disk will be more likely to cause density enhancements and "light up" the disk than a kick perpendicular to it. The kick direction can be clearly important on larger scales, as well, and affect phenomena that would occur on long timescales ( $\gg$ years) after the merger. For example, the angle relative to a large-scale galactic disk can determine whether a kicked BHB ends up outside the galaxy or not, and perhaps also whether shocks are produced when a BHB plunges into such a large-scale disk. While these issues merit investigation, in this Letter we focus on the smaller scales and shorter timescales ( $\lesssim$ months) that are relevant to prompt LISA counterparts.

Schnittman \& Buonanno (2007; hereafter SB07) used the "effective one-body approach" and derived a scaling formula that yields the recoil velocity vector for arbitrary mass ratios and spin vectors. While their results have not been tested for generic spins, they agree well (to within 20-30\%) with numerical results in those special configurations where they were tested (including numerical calculations for configurations with the spins parallel or at intermediate angles with respect to the orbital plane; see Campanelli et al. 2007a,b, González et al. 2007a; Herrmann et al. 2007b, Tichy \& Mar- 
ronetti 2007).

In this Letter, we investigate the response of a circumbinary disk to the kick, by following the perturbed, Keplerian orbits of collisionless massless test particles around the recoiling BHB. The purpose of this exercise is to demonstrate that prompt shocks or strong density enhancements are likely to arise when the kick is aligned with the plane of the circumbinary disk, whereas they may be less likely for highly inclined kicks. We will then use the formula of SB07 for the dependence of the kick speed and direction on the mass ratio and spins of the BHs, to argue that a significant fraction ( $\gtrsim$ few $\%$ ) of kicks may be both sufficiently large and well aligned with the orbital plane for strong shocks to be produced within a few weeks after coalescence.

\section{CIRCUMBINARY DISKS}

We begin with the assumption that the kicked BHB is surrounded by a rotationally supported, geometrically thin gaseous circumbinary disk. The idealized case of a smooth axisymmetric accretion disk, aligned with the binary's orbital plane (Bardeen \& Peterson 1975), with a simple vertical structure can be described by its density and temperature profiles $(\rho(r), T(r))$, as well as the scale height $H(r)$. This minimal information is needed (i) to compute the angle extended by the disk as viewed from the center, and (ii) to examine whether the kick is supersonic ( $v_{\text {kick }}>c_{s}$; a necessary condition for shocks to occur). In addition, we need to know the overall disk size (necessary to assess what fraction of the disk gas remains bound to the recoiling $\mathrm{BHB}$ ), and whether the disk mass is comparable to the BHB mass (necessary to assess whether the BHB is slowed down by the disk).

Unfortunately, fully self-consistent and stable accretion disk models around supermassive BHs are both difficult to produce, and require many ad-hoc assumptions (see, e.g., the review by Blaes 2007 for a list). While variants of the parametric so-called $\alpha$-disk models (Shakura \& Sunyaev 1973) have been successfully calibrated against observations of stellar-mass objects, observations of active galactic nuclei (AGN) have not led to similarly robust constraints on disk models around supermassive BHs. Nevertheless, several authors have discussed the possible behavior of a circumbinary $\alpha$-disk (e.g. Armitage \& Natarajan 2002; Milosavlievic \& Phinney 2005; Dotti et al. 2006). A key quantity is the critical orbital semi-major axis $a_{\text {crit }}$ at which the time-scale for the decay of the orbit due to gravitational radiation becomes shorter than the time-scale for viscous evolution (Begelman et al. 1980). Once the binary separation shrinks below this value at $t_{\text {crit }}$, the gas outside will not evolve significantly before the BHs coalesce. Most of the "kick" momentum is accumulated during the final stages of coalescence, on a rapid time-scale (Schnittman \& Buonanno 2007), hence a snapshot of the disk at $t_{\text {crit }}$ outside $\sim a_{\text {crit }}$ should still describe the disk around the kicked binary. Prior to $t_{\text {crit }}$, the torques from the binary may create a central cavity nearly devoid of gas within the radius $r_{\text {cavity }} \sim 2 a_{\text {crit }}$ (for a nearly equal-mass binary, e.g. Artymowicz \& Lubow 1994; MacFadyen \& Milosavljević 2006), or a narrower gap around the orbit of the lower-mass $\mathrm{BH}$ in the case of unequal masses $q \equiv M_{1} / M_{2} \ll 1$ (e.g. Armitage \& Natarajan 2002). In the latter case, the lower-mass hole will "usher" the gas inward as its orbit decays, so by the time of coalescence, this gas may accrete onto the more massive $\mathrm{BH}$, again leaving a nearly empty central cavity of radius $a_{\text {crit }}$.

In the inner regions of a disk around a supermassive $\mathrm{BH}$, the dominant vertical support is expected to be radiation pressure (as opposed to gas pressure), and electron scattering dominates the opacity over absorption (e.g. Shapiro \& Teukolsky 1983, p. 440). Milosavljevic \& Phinney (2005) presented an explicit model for a circumbinary disk with a central cavity, for the case of a nearly equal-mass binary at $t_{\text {crit }}$. They find, under the above conditions for the inner disk $\left(r_{\text {cavity }}=2 a_{\text {crit }}\right)$,

$$
r_{\text {cavity }}=117 r_{\mathrm{S}} \alpha_{-1}^{-0.34}\left(\eta_{-1} / \dot{m}\right)^{0.24} M_{6}^{0.08}\left[4 q /(1+q)^{2}\right]^{0.42},
$$

where $M_{6}$ denotes the total BHB mass $M_{1}+M_{2}$ in units of $10^{6} \mathrm{M}_{\odot}, r_{\mathrm{S}}=2 G M / c^{2}$ is the Schwarzschild radius, $0.1 \alpha_{-1}$ is the effective $\alpha$-parameter relating the kinematic viscosity to the gas (not total) pressure, $\dot{m}$ is the mass accretion rate at $r_{\text {cavity }}$ in units of the Eddington rate $\dot{M}_{\text {Edd }}$ (i.e. the accretion rate that would produce the Eddington luminosity with the radiative efficiency $\left.0.1 \eta_{-1}\right)$, and $q=M_{1} / M_{2} \leq 1$ is the mass ratio (note that we neglect here the dependence on additional dimensionless coefficients of order unity).

The thickness of the disk at its inner edge $r_{\text {cavity }}$ is given by

$$
\frac{h}{r}=0.46 M_{6}^{-0.12}\left[4 q /(1+q)^{2}\right]^{-1.84} \alpha_{-1}^{0.76}\left(\dot{m} / \eta_{-1}\right)^{2.43}
$$

and the mid-plane gas temperature is

$$
T=1.7 \times 10^{6} M_{6}^{-0.28}\left[4 q /(1+q)^{2}\right]^{-0.49} \alpha_{-1}^{0.19}\left(\dot{m} / \eta_{-1}\right)^{0.86} \mathrm{~K} .
$$

The ratio of radiation and gas pressure is given by

$$
\beta \equiv \frac{P_{\text {rad }}}{P_{\text {gas }}}=2600 M_{6}^{-0.04}\left[4 q /(1+q)^{2}\right]^{-1.84} \alpha_{-1}^{1.67}\left(\dot{m} / \eta_{-1}\right)^{4.25} .
$$

Note that the disk is marginally thin at $r_{\text {cavity. The expected }}$ radial profile of such a disk outside $r_{\text {cavity }}$ was discussed by Goodman \& Tan (2004). In the stable case considered by Milosavljevic \& Phinney (2005), when the viscosity is proportional to the gas (rather than the total) pressure, the midplane temperature and density, and surface density vary with radius as $T \propto r^{-9 / 10}, \rho \propto r^{-3 / 5}$, and $\Sigma \propto r^{-3 / 5}$. The ratio of gas/total pressure varies as $\beta \equiv P_{\text {gas }} / P_{\text {tot }} \propto r^{21 / 10}$ (in the limit of $\beta \ll 1$ ). Inside the transition radius where $\beta \approx 1$,

$$
r_{\text {tr }} \approx 1.9 \times 10^{3} M_{6}^{2 / 21} \alpha_{\text {tot }-1}^{2 / 21}\left(\dot{m} / \eta_{-1}\right)^{16 / 21} r_{\mathrm{S}},
$$

the scale height $h$ is constant, or $h / r \propto 1 / r$ (here $\alpha_{\text {tot,-1 }}$ relates the kinematic viscosity to the total pressure). This further assumes that the disk is optically thick. Beyond $r_{\text {tr }}$, gas pressure becomes dominant for vertical support, and the scaleheight begins to rise as $h \propto r^{21 / 20}$. Somewhat farther out, beyond $\sim 10,000 r_{\mathrm{S}}$ (for $M_{6} \sim 1$ ), the disk will become selfgravitating and subject to Toomre-instability. There are both theoretical and observational reasons to suspect that disks in real AGN extend to larger radii (Goodman 2003; Blaes 2007). However, note that the orbital time-scale at this radius is $\approx 2$ years, so that in the context of prompt LISA counterparts, we are not interested in radii outside this annulus.

To summarize, based on the above, we adopt the following simplified picture for the disk around a fiducial $M_{1}+M_{2}=$ $10^{6} \mathrm{M}_{\odot}$ binary. The disk has an inner edge at $100 r_{\mathrm{S}}$ (inside which it is empty) and an outer edge at $10,000 r_{\mathrm{S}}$ (outside which there may still be gas, but it evolves slowly and we do not follow it). The scale-height and temperature at the inner edge is $h / r=0.46$ and $T=1.7 \times 10^{6} \mathrm{~K}$, respectively. The scale-height remains constant with radius out to $2,000 r_{\mathrm{S}}$, be- 


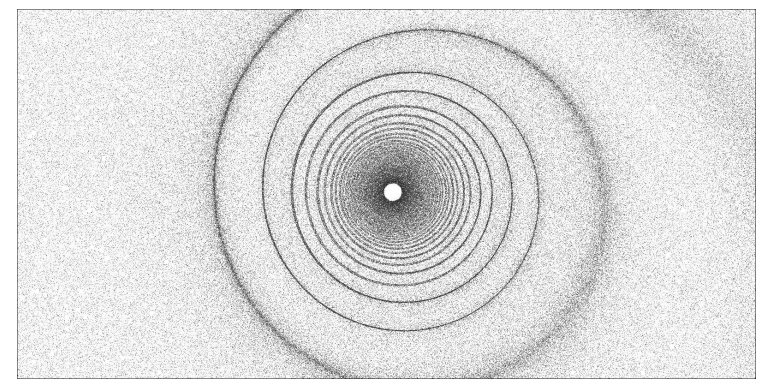

FIG. 1.- The top view of a disk around an $M_{1}+M_{2}=10^{6} \mathrm{M}_{\odot}$ BH binary, which recoiled within the disk plane at the velocity of $v_{\text {kick }}=500 \mathrm{~km} \mathrm{~s}^{-1}$, oriented vertically upward in the diagram. The disk is initially assumed to have an inner edge at $r_{\mathrm{in}}=100 r_{\mathrm{S}}$, and is shown here out to a radius of $r_{\mathrm{in}}=5,000 r_{\mathrm{S}}$, at a time $t=90$ days after the kick. A similar tightly wound spiral caustic patterns develops already in less than a month. The dark/light shades correspond to regions of high/low density, the low-density regions being similar to the initial surface density, and the high-density regions about 10 times overdense.

yond which it increases nearly linearly $\left(h \propto r^{21 / 20}\right)$. The temperature varies with radius as $T \propto r^{-9 / 10}$.

The important features of such a disk (as well as other proposed variants of $\alpha$-disks) are the following: (i) orbital motions in thin disks are supersonic (Pringle 1981), so that the gas is susceptible to shocks if disturbed; (ii) at the relevant radii outside $100 r_{\mathrm{S}}$ the viscous time-scale is long, so that the orbits are near Keplerian; (iii) gas near the inner edge of disk is tightly bound to the kicked BHB ( $\left.v_{\text {orbit }} \sim 3 \times 10^{4} \mathrm{~km} / \mathrm{s}\right)$, but the outer edge $\left(v_{\text {orbit }} \sim 3 \times 10^{3} \mathrm{~km} / \mathrm{s}\right)$ can be marginally bound, or even unbound, for large kicks (the approximate condition for being bound is $v_{\text {orbit }} \gtrsim 2.4 v_{\text {kick }}$ ), and (iv) the total disk mass within $10,000 r_{\mathrm{S}}$ is much less than the BHB mass, which justifies ignoring the inertia of the gas bound to the BHB.

\section{THE RESPONSE OF THE DISK TO KICKS}

For a quantitative assessment of the disk's response to the kick, we employ the following approximation: the disk particles are assumed to be massless, collisionless, and initially on co-planar, circular orbits. The kick simply adds the velocity $\vec{v}_{\text {kick }}$ to the instantaneous orbital velocity of each particle (in the inertial frame centered on the BHB). We used $N=10^{6}$ particles, distributed randomly and uniformly along the twodimensional surface of the disk. The kick velocity was varied between $500 \mathrm{~km} \mathrm{~s}^{-1}<v_{\text {kick }}<4,000 \mathrm{~km} \mathrm{~s}^{-1}$, and directed either perpendicular or parallel to the initial disk plane.

Figure 1 shows, as an example, a face-on view of the surface density of the disk 90 days after a kick with $v_{\text {kick }}=$ $500 \mathrm{~km} \mathrm{~s}^{-1}$ in the plane of the disk $\left(i_{\text {kick }}=0^{\circ}\right)$. The sharp, tightly wound spiral features clearly seen in the figure trace the locus of points where particles cross each other, corresponding formally to a density caustic. The spiral caustic first forms at $\sim 30$ days, and then propagates outward at a speed of $\approx 500 \mathrm{~km} \mathrm{~s}^{-1}$. This behavior can be roughly understood as follows: at a given radius $r_{c}$, the caustic forms at the time $t_{\mathrm{c}}$ when the radial epicyclic motions from two neighboring annuli, separated by the epicyclic amplitude $\sim\left(v_{\text {kick }} / v_{\text {orbit }}\right) r_{c}$, overlap (this predicts $r_{c} \sim t_{\mathrm{c}} v_{\text {kick }}$ ). We also found that as $v_{\text {kick }}$ is increased, the spiral caustics spread farther out. Eventually, for kicks strong enough so that a significant fraction of the disk is unbound, the caustics loose their coherent spiral patterns, and develop complex two-dimensional shapes.

Figure 2 shows a side view of the 3D particle density one week after a kick with the velocity $v_{\text {kick }}=500 \mathrm{~km} \mathrm{~s}^{-1}$ perpendicular to the disk $\left(i_{\text {kick }}=90^{\circ}\right)$. The density profile in this

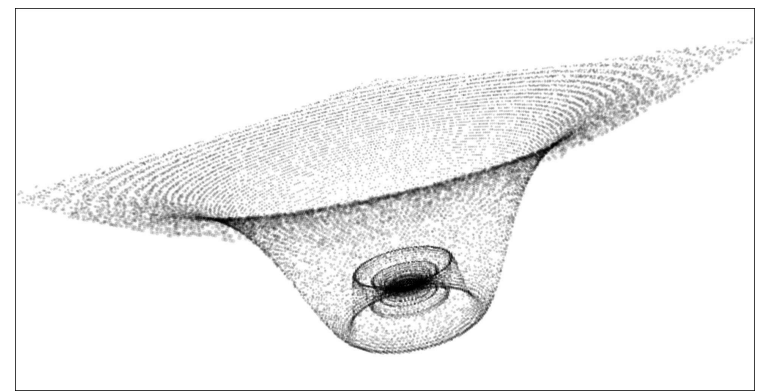

FIG. 2.- The aerial view of a disk as in Figure 1 except here the kick is oriented perpendicularly to the disk, and the snapshot is taken at $t=1$ week. For visual clarity, the graphic contrast was increased relative to Fig. 1 and the diagram stretched by a factor of 10 along the axis perpendicular to the disk plane. The density distribution is azimuthally symmetric, and while there are mild concentric density fluctuations, strong enhancements (i.e. caustics) develop only after a delay of $\approx$ one year.

case remains azimuthally symmetric, but still develops concentric rings of density fluctuations. The important difference from the parallel kick case is that the density enhancements are much weaker (at the ten percent level). By examining the time-evolving radial cross-sections, we have verified that sharp density enhancements, i.e. true caustics caused by the orbit-crossing of particles, first appear only after one year, and involve a smaller fraction of the disk particles.

More generally, kicks can be expected to occur in a direction intermediate between the two extremes shown in Figs. 1 and2 Following a kick at an arbitrary inclination angle, each particle still remains on a Kepler orbit. Each new orbit will follow an ellipse in a plane that is tilted by an angle of order between $\pm v_{\text {kick }} / v_{\text {orbit }}$ about the axis connecting the instantaneous position of the particle with the central BHB. Hence, after an orbital time, the particles of the disk will be smeared vertically, effectively thickening the original disk. The twodimensional simulations we performed do not conclusive tell us a critical kick inclination angle for prompt density caustics to be produced - this would require three-dimensional simulations, following the orbits of particles in a 3D disk of finite thickness. However, since the prompt, strong density enhancements appear to develop in the plane of the kick, near the inner edge of the disk, one may conjecture that this critical inclination angle is given roughly by $i_{\text {kick }} \lesssim \arctan (h / r)$ (with $h / r=0.46$ evaluated at $r_{\text {cavity }}$ ).

In Figure 3, we show the distribution of kick angles, taken from SB07, assuming that both spins are randomly oriented. We require further that the component of $v_{\text {kick }}$ within the orbital plane exceed $3-500 \mathrm{~km} \mathrm{~s}^{-1}(\approx 3-5 \times$ the sound speed; see justification in $\S$ (4) below). A strong kick within the orbital plane is produced if the spins are large and parallel to the angular momentum, but anti-aligned with each other.

The main conclusion to draw from Figure 3 is that causticproducing kicks are not rare - in the cases shown in the panels, they occur at least a few percent of the cases. This conclusion remains true unless (i) the spins of the BHs is significantly below the maximal Kerr value $(a \lesssim 0.9)$, or (ii) the spins are significantly aligned with each other. The latter may be expected if the spin of both $\mathrm{BHs}$ results from accretion from the same disk (Bogdanović et al. 2007).

The criteria defined above for producing caustics may turn out very conservative. For example, prompt caustics could develop when particles from different layers of the disk cross along their orbits. A plausible weaker criterion for shocks is that the kick-induced tilts of the orbits do not thicken the disk 

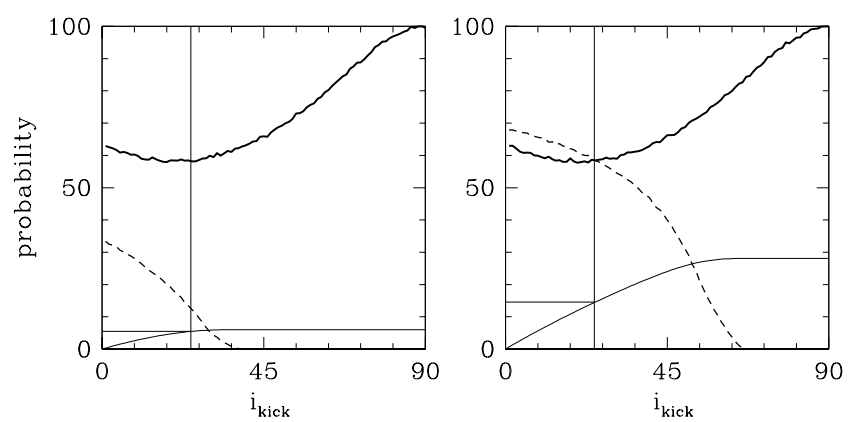

FIG. 3.- The thick solid curves in both panels show the (arbitrarily normalized) differential probability distribution of kick inclinations relative to the disk plane, using the SB07 formula to compute $\vec{v}_{\text {kick }}$. Both panels assume an equal-mass binary ( $q=1$; we find results changed only by a few percent for $q=0.1$ ). We assume random spin orientations. On the left panel we assume dimensionless spins of $a_{1}=a_{2}=1$, while on the right panel $a_{1}=a_{2}=0.9$. The dashed curves show the fraction of kicks at a given inclination angle that have a component in the disk exceeding a fixed threshold ( $v_{\text {kick, } \|} \geq 500 \mathrm{~km} \mathrm{~s}^{-1}$ in the left panel, and $300 \mathrm{~km} \mathrm{~s}^{-1}$ in the right panel). The thin curves indicate the cumulative probability of sufficiently fast kicks with inclinations below a given value; the horizontal line shows the fraction of such kicks with inclinations $\leq 24^{\circ}$ ( 6 and $15 \%$ percent in the left and right panels).

beyond its original scale-height. Apart from trigonometric factors of order unity that describe its azimuthal dependence, the tilt angle is given by $i_{\text {tilt }} \sim\left(v_{\text {kick }} / v_{\text {orbit }}\right) i_{\text {kick }}$; requiring only $i_{\text {tilt }} \lesssim \arctan (h / r)$, or $i_{\text {kick }} \lesssim\left(v_{\text {orbit }} / v_{\text {kick }}\right) \arctan (h / r)$, density caustics could arise even for nearly perpendicular kicks. In follow-up work, we will employ three-dimensional simulations, to clarify the relevant caustic formation criterion.

\section{DISCUSSION}

The main result of this Letter is that strong density enhancements can form promptly after a supersonic kick in the plane of the circumbinary disk, within a few weeks of the coalescence of $\mathrm{a} \sim 10^{6} \mathrm{M}_{\odot}$ BHB. Because the disk is cold, and caustics (Fig. 1) are formed when particles first cross each other along their orbits, this implies that corresponding shocks could occur in a gas disk. For hydrodynamical shocks to occur within a finite-pressure gas, the relative motions $v_{\mathrm{c}}$ between the neighboring particles that produce the caustic must exceed the sound speed. At the outermost radius where the disk is marginally bound to the BHB, one expects $v_{\mathrm{c}} \sim v_{\text {kick }} \sim v_{\text {orbit }}$; relative motions will be slower further inside. The relative speed should roughly correspond to covering the epicyclic amplitude $\sim\left(v_{\text {kick }} / v_{\text {orbit }}\right) r_{c}$ in the caustic-formation time $t_{\mathrm{c}} \sim$ $r_{c} / v_{\text {kick }}$, yielding $v_{c} \sim v_{\text {kick }}^{2} / v_{\text {orbit }}$. For $v_{\text {kick }}=500 \mathrm{~km} \mathrm{~s}^{-1}$, this predicts $v_{\mathrm{c}} \sim 25 \mathrm{~km} \mathrm{~s}^{-1}\left(r / 1000 r_{\mathrm{S}}\right)^{1 / 2}$; we have verified in our simulations that particles cross the caustics with speeds at about $\sim 30 \%$ above this predicted value. Compared with the sound speed $c_{s} \approx 25 \mathrm{~km} \mathrm{~s}^{-1}\left(r / 1000 r_{\mathrm{S}}\right)^{-9 / 20}$, this suggests that the density waves produced by the kick in the gas beyond $\sim 700 r_{\mathrm{S}}$ will indeed steepen into shocks. We also found that the inclination of the kick may be important in determining the strength and timing of such shocks - perpendicular kicks would only produce weaker density enhancements, at least until a delay of about a year. A non-negligible fraction ( $\gtrsim$ several \%) of kicks could, however, be sufficiently large and well aligned with the orbital plane for shocks to be produced within a few weeks after coalescence.

The nature of the emission resulting from the shocks or density enhancements will have to be addressed in future work, by computing the heating rate at the spiral shocks, and modeling the overall disk structure and vertical radiation transport. However, the disk's luminosity resulting from the kick, $L_{\text {kick }}$ could be a non-negligible fraction of the BHB's Eddington luminosity, and therefore potentially observable (Kocsis et al. 2007b). If $M_{\text {shock }}=f_{\text {shock }}\left(M_{1}+M_{2}\right)$ is the mass of the shocked gas that is heated to temperatures corresponding to $v_{\text {shock }}$, and $t_{\text {shock }}$ is the time-scale on which the corresponding thermal energy is converted to photons, then $L_{\text {kick }} \approx(1 / 2) M_{\text {shock }} v_{\text {shock }}^{2} / t_{\text {shock}}$, and $L_{\text {kick }} / L_{\text {Edd }} \approx$ $0.1\left(f_{\text {shock }} / 10^{-5}\right)\left(v_{\text {shock }} / 500 \mathrm{~km} \mathrm{~s}^{-1}\right)^{2}\left(t_{\text {shock }} / 1 \text { month }\right)^{-1}$. We may also speculate on the spectral evolution of the "kick after-glow". Assuming $M_{\text {shock }} \propto \Sigma r d r \propto r^{19 / 10}$ (with $\Sigma \propto$ $r^{-3 / 5}$ and $d r \propto r^{1 / 2}$, the epicyclic amplitude), and $v_{\text {shock }} \approx v_{c}$, we find $L_{\text {kick }} \propto r^{24 / 10}$. This suggests that the luminosity may be dominated by the outermost shocked shells. The spectrum will then peak at the characteristic photon energy corresponding to $k T_{\text {shock }} \propto v_{\mathrm{c}}^{2} \propto v_{\text {orbit }}^{-2} \propto r$. The shocks could therefore result in an afterglow, starting from $r_{\text {cavity }} / v_{\text {kick }} \sim 30$ days, first peaking in the UV band $(\sim 10 \mathrm{eV})$, and then hardening to the soft $\mathrm{X}$-ray $(\sim 100 \mathrm{eV})$ range after one year. The detection of such an afterglow would help identify EM counterparts to GW sources discovered by LISA.

Our results need to be verified in three-dimensional simulations that resolve the orbits within a finite disk thickness. A realistic disk model, incorporating gas dynamics, is needed to study the correspondence between collisionless caustics and gaseous shocks. Finally, the SB07 formula needs to be confirmed for generic spin configurations. Nevertheless, our results do suggest that kicks due to gravitational waves may produce a prompt EM signal.

We thank A. Buonanno, M. Milosavljević, K. Menou, and B. Kocsis for useful discussions, and M. Milosavljević for suggesting the arguments based on epicycles. This work was supported by the Polányi Program of the Hungarian National Office for Research and Technology (NKTH) and by NASA grant NNG04GI88G (to ZH).

\section{REFERENCES}

Armitage, P. J., \& Natarajan, P. 2002, ApJ, 567, L9

Artymowicz, P., \& Lubow, S. H. 1994, ApJ, 421, 651

Baker, J. G., Centrella, J., Choi, D.-I., Koppitz, M., van Meter, J. R., \& Miller, M. C. 2006, ApJ, 653, L93

Baker, J. G., Boggs, W. D., Centrella, J., Kelly, B. J., McWilliams, S. T., Miller, M. C., \& van Meter, J. R. 2007, ApJ, 668, 1140

Bardeen, J. M., \& Peterson, J. A. 1975, ApJ, 195, L65

Begelman, M. C., Blandford, R. D., \& Rees, M. J. 1980, Nature, 287, 307

Blaes, O. 2007, in "The Central Engine of Active Galactic Nuclei", ed. L. C.

Ho and J.-M. Wang, (San Francisco: ASP), e-print astro-ph/0703589

Bogdanović, T., Reynolds, C. S., \& Miller, M. C. 2007, ApJ, 661, L147

Campanelli, M., Lousto, C. O., Zlochower, Y., \& Merritt, D. 2007a, ApJ, 659, L5 2007b, Phys. Rev. Lett, 98, 231102

Dotti, M., Salvaterra, R., Sesana, A., Colpi, M., \& Haardt, F. 2006, MNRAS, 372, 869

González, J. A., Sperhake, U., Brügmann, B., Hannam, M. D., \& Husa, S. 2007a, Phys. Rev. Lett., 98, 091101

González, J. A., Hannam, M. D., Sperhake, U., Brügmann, B., \& Husa, S. 2007b, Phys. Rev. Lett, 98, 231101

Goodman, J. \& Tan, J. C. 2004, ApJ, 608, 108

Herrmann, F., Hinder, I., Shoemaker, D., Laguna, P., \& Matzner, R. A.

2007a, ApJ, 661, 430

2007b, Phys. Rev. D, 76, 084032

Herrmann, F., Hinder, I., Shoemaker, D., \& Laguna, P. 2007c, Class. Quant.

Gr., 24, 33 
Kocsis, B., Haiman, Z., Menou, K., \& Frei, Z. 2007a, Phys. Rev. D, 76, 022003

Kocsis, B., Haiman, Z., \& Menou 2007b, ApJ, submitted, e-print arXiv:0712.1144

Koppitz, M., Pollney, D., Reisswig, C., Rezzolla, L., Thornburg, J., Diener, P., \& Schnetter, E. 2007, Phys. Rev. Lett. 99, 041102

Lang, R. N., \& Hughes, S. A. 2007, ApJ, submitted, e-print arXiv:0710.3795

Loeb, A. 2007, Phys. Rev. Lett. 99, 041103

MacFadyen, A., \& Milosavljević, M. 2006, ApJ, in press, e-print astro-ph/0607467

Madau, P., \& Quataert, E. 2004, ApJ, 606, L17

Merritt, D., Milosavljevic, M., Favata, M., Hughes, S. A., \& Holz, D. E. 2004, ApJ, 607, L9

Milosavljevic, M., \& Phinney, E. S. 2005, ApJ, 622, L93

Pringle, J. E. 1981, ARA\&A, 19, 137
Schnittman, J. D., \& Buonanno, A. 2007, ApJ, 662, L63

Schnittman, J. D., Buonanno, A., van Meter, J. R., Baker, J. G., Boggs, W. D., Centrella, J., Kelly, B. J., \& McWilliams, S. T. 2007, Class. Quant. Gr., submitted, e-print arXiv:0707.0301

Shakura, N. I., \& Sunyaev, R. A. 1973, A\&A, 24, 337

Shapiro, S. L. 2005, ApJ, 620, 59

Shapiro, S. L., \& Teukolsky, S. A. 1983, "Black Holes, White Dwarfs, and Neutron Stars: The Physics of Compact Objects" (John Wiley \& Sons: New York).

Tichy, W. \& Marronetti, P. 2007, Phys. Rev. D, 76, 1502

Volonteri, M., \& Perna, R. 2005, MNRAS, 358, 913

Yoo, J., \& Miralda-Escudé, J. 2004, ApJ, 614, L25 